\title{
Structural properties of embedded Ge nanoparticles modified by swift heavy-ion irradiation
}

\author{
L. L. Araujo, ${ }^{1, *}$ R. Giulian, ${ }^{1}$ D. J. Sprouster, ${ }^{1}$ C. S. Schnohr, ${ }^{1}$ D. J. Llewellyn, ${ }^{1}$ B. Johannessen, ${ }^{2}$ \\ A. P. Byrne, ${ }^{3}$ and M. C. Ridgway ${ }^{1}$ \\ ${ }^{1}$ Department of Electronic Materials Engineering, Research School of Physics and Engineering, Australian National University, \\ Canberra, Australian Capital Territory 0200, Australia \\ ${ }^{2}$ Australian Synchrotron, 800 Blackburn Road, Melbourne, Victoria 3168, Australia \\ ${ }^{3}$ Department of Nuclear Physics, Research School of Physics and Engineering, Australian National University, Canberra, Australian Capital \\ Territory 0200, Australia
}

(Received 20 December 2011; revised manuscript received 24 April 2012; published 7 June 2012)

\begin{abstract}
Silica-embedded Ge nanoparticles (NPs) of different sizes irradiated with swift heavy ions (SHIs) at a given energy may reportedly elongate along the incident ion direction, perpendicular to it, or not at all. Here, for a given NP size distribution, we have investigated the SHI energy dependence of the elongation process. Higher-energy irradiation generally yielded elongation along the ion track (as previously observed), but for lower-energy irradiation, elongation both parallel and perpendicular to the ion direction was observed. We demonstrate that NP size and electronic energy loss together govern the elongation process, reinforcing the proposed model where elongation perpendicular to the ion direction is only expected for Ge NPs bigger than the mean ion track diameter in silica. Here, a wide fluence range is also probed, enabling us to follow in more detail the transition from spherical unirradiated Ge NPs to Ge NPs elongated either parallel or perpendicular to the ion beam. X-ray absorption spectroscopy (XAS) measurements are utilized for the quantification of crystalline, amorphous, and oxidized environments around Ge atoms. Combining such results with transmission electron microscopy (TEM) observations shows the Ge NPs are rendered amorphous prior to elongation, potentially via a melt-and-quench process. Thereafter, stronger electron-phonon coupling in amorphous Ge compared to crystalline Ge may potentially influence the elongation process. The Ge NP amorphization occurs at lower fluences for higher irradiation energies, indicating electronic energy loss—and not ballistic effects—governs the amorphization. Subsequent to amorphization and elongation, TEM and XAS results also show the NPs gradually intermix with $\mathrm{SiO}_{2}$ and dissolve within the matrix as the irradiation fluence increases. We discuss the impact of such results in the ion beam tailoring of Ge NPs for technological applications.
\end{abstract}

DOI: 10.1103/PhysRevB.85.235417

PACS number(s): 64.70.Nd, 61.46.-w

\section{INTRODUCTION}

The modification of micro- and nanostructures by ion beams ${ }^{1-4}$ has attracted considerable attention in recent years. In principle, ion irradiation can be used to tailor desired properties in a controlled manner, thus producing improved systems for application in electronic, photonic, and memory devices. Swift heavy-ion irradiation (SHII) of nanostructures, in particular, has been shown to induce changes in size and shape $^{4-12}$ as well as crystallographic phase. ${ }^{13}$ Such changes have a direct impact on the optical and magnetic properties of the irradiated systems.

Previous studies on SHII of crystalline Ge nanoparticles (NPs) embedded in silica $\left(\mathrm{a}-\mathrm{SiO}_{2}\right)$ have shown that their shape can be modified by SHII in different ways. Depending on the initial NP size, elongation along the ion beam direction, perpendicular to it, or no elongation at all were observed for the same irradiation energy and fluences. ${ }^{11,12}$ That is in contrast to most studies on SHII of embedded metallic NPs ${ }^{5-9,14-16}$ performed with different ions at a few hundred MeVs, where no elongation was observed for NP sizes below a threshold diameter, and only elongation along the beam direction was apparent. In a recent report, it was shown that SHII of Au NPs with very high-energy ( $5 \mathrm{GeV}$ ) $\mathrm{Pb}$ ions (electronic energy loss of $17 \mathrm{keV} / \mathrm{nm}$ in silica) resulted not only in the formation of nanorods elongated along the ion beam direction, but also in the formation of faceted structures. ${ }^{4}$

Although the initial NP size clearly plays a role in the deformation process, the ion and matrix species, the irradiation energy and fluence (or alternatively the electronic stopping power and the total deposited energy), and the sample temperature are equally important. ${ }^{1,17}$ The ion flux density during irradiation was shown to have a strong effect on the elongation of $\mathrm{Au}$ nanodisks embedded in $\mathrm{a}-\mathrm{SiO}_{2}$, with an increase in flux density resulting first in more pronounced elongation and then into dissolution of the Au nanodisks. ${ }^{18}$ This was ascribed to a global increase in sample temperature due to many simultaneous ion impacts, potentially increasing the local temperature above that required for the vaporization of $\mathrm{a}_{-} \mathrm{SiO}_{2} .{ }^{18} \mathrm{~A}$ study on the combined effects of SHII energy and fluence on Au NPs of different sizes embedded in $\mathrm{a}-\mathrm{SiO}_{2}$ was reported in Ref. 17. The existence of a threshold fluence and of a saturation fluence for NP elongation, both dependent on NP size and irradiation energy, were verified. The threshold fluence decreased with increasing irradiation energy and decreasing NP size. The NP concentration was also shown to influence the elongation process, with NP dissolution and the formation of very long nanorods composed of material from more than a single initial NP being observed. ${ }^{17}$ Similar findings were also reported for SHII of Pt NPs embedded in $\mathrm{a}_{-} \mathrm{SiO}_{2} .{ }^{10}$

By comparing SHII of silica-embedded and freestanding Au NPs under the same conditions, van Dillen et al. ${ }^{3}$ have shown that an encapsulating matrix where ion tracks are formed is required for the deformation of metallic NPs. No appreciable deformation was observed for $\mathrm{Pb}$ and $\mathrm{Bi}$ NPs embedded in a crystalline matrix (Al) submitted to SHII with $30 \mathrm{MeV} \mathrm{C}_{60}$ clusters, ${ }^{19}$ reinforcing the fact that the formation 
of ion tracks in the matrix is necessary for NP elongation. More recent results on the SHII of Sn NPs localized at the interface between $\mathrm{a}-\mathrm{SiO}_{2}$ and c-Si layers clearly demonstrated $\mathrm{NP}$ elongation only takes place towards the a-SiO${ }_{2}$ layer, where ion tracks are formed. ${ }^{20}$ On the other hand, freestanding $\mathrm{ZnS}$ (semiconductor) microstructures were shown to deform under irradiation with $4 \mathrm{MeV}$ Xe ions, elongating perpendicularly to the incident beam (as also verified for silica colloids). This result implies that amorphous NPs, unlike metallic NPs, may elongate under SHII without the presence of an embedding matrix. Results on the $30 \mathrm{MeV} \mathrm{Cu}$ irradiation of $\mathrm{Si}$ micropillars (1.5 $\mu \mathrm{m}$ tall $)$ have shown that crystalline Si pillars did not deform plastically, unlike amorphous $\mathrm{Si}$ pillars, ${ }^{21}$ demonstrating the response of semiconductor microstructures to SHII depends on their structural phase.

The deformation of embedded nanostructures may be constricted by the surrounding matrix. Most studies on embedded NPs invoke the thermal spike (TS) model $^{4-9,12}$ to explain the observed NP deformation under SHII. The thermal spike model considers that the energy lost by the impinging ions to the target electrons is subsequently transferred to the lattice through electron-phonon interactions. ${ }^{8}$ This increases the vibrational amplitude of the atoms in the target or the lattice temperature. If enough energy is transferred to surpass the melting point of the target material, then ion tracks can be formed in the matrix by fast cooling of the molten region. If enough energy is transferred to surpass the melting point of the target material, a molten ion track is formed. Nanoparticle elongation may result from molten nanoparticle flow within such a track, potentially under the influence of large shear stresses. The peculiar behavior of Ge NPs, which elongate perpendicular to the beam direction, has previously been assigned to the correlation between NP and ion track diameters (with the former bigger than the latter for perpendicular elongation) as well as to the fact that bulk Ge presents a volume contraction of about $5 \%$ upon melting. ${ }^{12}$

This work aims at further advancing the current knowledge about the effects of SHII on embedded Ge NPs by probing not only the initial (spherical NPs) and final (elongated NPs) endpoints of the process, but considering the intermediate steps in a wider fluence range than previous works. ${ }^{11,12}$ The same NP size distribution was irradiated with different energies, and structural properties were correlated to morphological changes by employing x-ray absorption spectroscopy (XAS) and transmission electron microscopy (TEM) measurements. Experimental details are given in Sec. II, followed by presentation of the results and discussion in Sec. III. The main findings are summarized in Sec. IV.

\section{EXPERIMENTAL DETAILS}

Ge NPs were formed in a $2-\mu \mathrm{m}$-thick a-SiO $\mathrm{Sitrix}_{2}$ by ion implantation, at liquid nitrogen temperature, of $1 \times 10^{17} \mathrm{~cm}^{-2}$ $\mathrm{Ge}$ ions at $2 \mathrm{MeV}$. The implantation was followed by thermal annealing at $1100{ }^{\circ} \mathrm{C}$ for $10 \mathrm{~h}$, under forming gas $\left(95 \% \mathrm{~N}_{2}+\right.$ $5 \% \mathrm{H}_{2}$ ). The implanted Ge peak concentration of $\sim 3$ at.\% was centered at a depth of $1.4 \mu \mathrm{m}$ inside the $\mathrm{SiO}_{2}$ layer. ${ }^{22}$ The NP diameter distribution was measured by transmission electron microscopy (TEM) and small-angle x-ray scattering (SAXS)



FIG. 1. (Color online) Size distribution measured by SAXS ${ }^{22}$ for the Ge NPs under study (bars). The vertical lines indicate the average track diameters for the irradiation energies listed above the top axis. The numbers beside the lines indicate the percentage of Ge NPs in the ensemble having diameter equal or smaller than the average track diameter for each energy.

and was centered at $\sim 9.0 \mathrm{~nm}$, as shown in Fig. 1. Further details can be found in Ref. 22 .

Swift heavy-ion irradiations were performed with the 14UD Pelletron accelerator at the Australian National University. All irradiations were performed at room temperature, with the sample stage cooled by a flowing hydrocarbon-based liquid. The beam was scanned over an area of $3 \times 6 \mathrm{~mm}^{2}$. Samples were irradiated in the fluence range of $4 \times 10^{10}-1 \times 10^{14} \mathrm{~cm}^{-2}$ with $\mathrm{Au}$ beams of 27, 54, and $185 \mathrm{MeV}$, for which the charge states of the impinging ions were $5+, 7+$, and $13+$, respectively. Stopping and Range of Ions in Matter (SRIM) ${ }^{23}$ was used to calculate the energy of the Au ions at the maximum of the $\mathrm{Ge}$ depth distribution in $\mathrm{a}-\mathrm{SiO}_{2}(1.4 \mu \mathrm{m})$. These values are listed in Table I, together with the corresponding electronic $\left(S_{e}\right)$ and nuclear $\left(S_{n}\right)$ energy losses for $\mathrm{Au}$ in both $\mathrm{a}-\mathrm{SiO}_{2}$ and $\mathrm{c}-\mathrm{Ge}$. The mean diameter of the tracks formed by the $\mathrm{Au}$ ions in the a- $\mathrm{SiO}_{2}$ matrix, as determined in Ref. 24, is also listed on Table I.

Cross-sectional TEM images were acquired in bright-field mode using a Philips CM300 microscope operating at $300 \mathrm{kV}$. Samples were prepared with a standard grinding/ion-beam milling protocol.

X-ray absorption spectroscopy (XAS) measurements at the Ge K-edge (11.103 keV) were performed at beamline 20-B of the Photon Factory, Japan. The measurement temperature was maintained at $15 \mathrm{~K}$ to minimize the thermal contribution to the disorder in the distribution of interatomic distances. Fluorescence spectra were recorded with a Ge pixel array detector. X-ray absorption spectroscopy samples were prepared according to the procedure outlined in Ref. 22. Bulk crystalline and amorphous Ge standards (c-Ge and a-Ge, respectively) were prepared by molecular-beam epitaxy and ion implantation, as described in Ref. 22. A crystalline $\mathrm{GeO}_{2}$ (c- $\mathrm{GeO}_{2}$ ) standard was prepared by fine grinding bulk c- $\mathrm{GeO}_{2}$ and mixing with boron nitride to a suitable dilution. 
TABLE I. Initial beam energy $E_{i}$, mean energy of Au ions at the Ge depth distribution maximum $E_{x}$, nuclear $\left(S_{n}\right)$ and electronic $\left(S_{e}\right)$ energy losses in c-Ge $\left(\rho=5.35 \mathrm{~g} / \mathrm{cm}^{3}\right)$ and a-SiO $2\left(\rho=2.20 \mathrm{~g} / \mathrm{cm}^{3}\right)$, and average track diameter in a-SiO $\mathrm{O}_{2}$ from Ref. 24.

\begin{tabular}{|c|c|c|c|c|c|c|}
\hline \multirow[b]{2}{*}{$E_{i}(\mathrm{MeV})$} & \multirow[b]{2}{*}{$E_{x}(\mathrm{MeV})$} & \multicolumn{2}{|c|}{$\begin{array}{l}\text { Energy loss in c-Ge } \\
\text { at } E_{x}(\mathrm{keV} / \mathrm{nm})\end{array}$} & \multicolumn{2}{|c|}{$\begin{array}{l}\text { Energy loss in a-SiO } \\
\text { at } E_{x}(\mathrm{keV} / \mathrm{nm})\end{array}$} & \multirow[b]{2}{*}{$D_{\text {track }}(\mathrm{nm})$} \\
\hline & & $S_{n}$ & $S_{e}$ & $S_{n}$ & $S_{e}$ & \\
\hline 27.0 & 20.5 & 1.3 & 4.0 & 0.8 & 3.6 & 5.0 \\
\hline 54.0 & 43.0 & 0.8 & 8.1 & 0.5 & 6.9 & 8.0 \\
\hline 185.0 & 162.7 & 0.3 & 23.2 & 0.2 & 16.0 & 11.0 \\
\hline
\end{tabular}

X-ray absorption spectroscopy spectra were averaged and energy calibrated using the AVERAGE program. ${ }^{25}$ Next, spectra were normalized using the ATHENA program, ${ }^{26}$ and linear combination fits were performed around the x-ray absorption near-edge structure (XANES) region for the Ge NPs samples. The c-Ge, a-Ge, and c-GeO ${ }_{2}$ spectra were used as standards to fit each Ge NP spectrum in the region from $30 \mathrm{eV}$ below to $100 \mathrm{eV}$ above the absorption edge. The extended $\mathrm{x}$-ray absorption fine structure (EXAFS) oscillations were extracted from the experimental spectra by background subtraction via the AUTOBK algorithm, as implemented in ATHENA. ${ }^{26}$ ATHENA and ARTEMIS $^{26}$ are graphical user interfaces developed to facilitate EXAFS data analysis with the IFEFFIT code. ${ }^{27}$ The signal from the c-Ge standard was used to refine the values of the energy shift parameter $\Delta E_{0}$ according to the procedure suggested in Ref. 28. This enabled the alignment of the wave number $k$ scale of the c-Ge theoretical standard generated by the FEFF $8.4^{29}$ code for all the samples, thus avoiding errors in the structural parameters due to a poor choice of the edge energy position $E_{0}$.

Structural parameters characterizing the interatomic distance distribution for the first-nearest neighbor (FNN) shell were determined with ARTEMIS ${ }^{26}$ for all samples. Data in $k$ space were Fourier-transformed with a Hanning window of width $0.50 \AA^{-1}$ and range 4.05-15.05 $\AA^{-1}$. The window defining the fitting region in the nonphase-corrected radial distance $R$ space was also of the Hanning type, with width $0.20 \AA$ and range from 1.60 to $2.70 \AA$. The FEFF8. 4 code $^{29}$ was used to calculate $a b$ initio effective scattering amplitudes and phase shifts. Each dataset was fitted simultaneously with multiple $k$ weightings of 1-3 to reduce correlations between the fitting parameters. The passive electron reduction factor $S_{0}{ }^{2}$ and the energy shift parameter $\Delta E_{0}$ were determined from the c-Ge data and kept constant when fitting the Ge NP samples. The obtained values were $S_{0}^{2}=0.98 \pm 0.07$ and $\Delta E_{0}=0.2 \pm$ $1.0 \mathrm{eV}$. Previous results have shown that the presence of asymmetry should always be taken into account for systems with some degree of structural disorder, such as bulk amorphous materials and nanoparticles, ${ }^{22}$ and thus the results presented herein correspond to fits including a third cumulant, the values of which were nearly constant for all irradiation conditions.

\section{RESULTS AND DISCUSSION}

\section{A. XANES results}

X-ray absorption near-edge structure spectra obtained for samples containing Ge NPs irradiated with representative fluences are shown in Figs. 2(a)-2(c) for irradiation energies of 27-185 MeV, respectively. Changes in spectra with increasing fluence are apparent for all energies, as indicated by the arrows. As discussed in a previous article reporting on the ion irradiation of Ge NPs with $5 \mathrm{MeV}$ Si ions (where no NP shape transformation was observed), such changes indicate amorphization of the NPs and the formation of Ge-O bonds. ${ }^{30}$ It is clear from Fig. 2 that, for any given fluence, the irradiation effect is more pronounced for the higher irradiation energies. $\mathrm{X}$-ray absorption near-edge structure spectra acquired for the c-Ge, a-Ge, and $\mathrm{c}-\mathrm{GeO}_{2}$ standards were identical to those presented in Ref. 30 and thus are omitted here for simplicity.

The fractions of Ge atoms in crystalline, amorphous, and oxidized environments were extracted from the XANES spectra through a linear-combination fitting procedure, previously described elsewhere. ${ }^{30}$ The results are presented in Fig. 3 for the three SHII energies. The unirradiated Ge NPs already contain an a-Ge fraction due to disorder at the NP surface/interface with the matrix, as previously verified by XAS measurements and molecular dynamics (MD) simulations. ${ }^{30,31}$ From the figure, it is clear that SHII causes a decrease in the c-Ge fraction accompanied by an increase in the a-Ge fraction, as well as the appearance of a $\mathrm{Ge}-\mathrm{O}$ fraction at higher irradiation fluences. A similar effect has been observed for Co NPs embedded in silica after irradiation with SHI. ${ }^{16}$ The vertical dash-dot lines in the figure indicate the fluences where the $\mathrm{c}-\mathrm{Ge}$ fraction has been reduced to $50 \%$, corresponding to $\sim 1.0 \times 10^{12}, 2.0 \times 10^{11}$, and $9.0 \times 10^{10} \mathrm{~cm}^{-2}$, respectively, for the energies of 27,54 , and $185 \mathrm{MeV}$. Since ballistic effects (nuclear energy loss) become more negligible as the irradiation energy increases, it is evident that amorphization of the Ge NPs and the formation of Ge-O bonds stem from electronic energy loss processes in the present study. The fraction of Ge atoms in an amorphous environment increases as the $\mathrm{c}-\mathrm{Ge}$ fraction decreases, but never reaches $100 \%$ due to the formation of $\mathrm{Ge}-\mathrm{O}$ bonds (by intermixing at the NP/matrix interface and gradual dissolution of the Ge NPs, as will be further discussed). Again, the effect is more pronounced for higher irradiation energies. Figures 3(a)-3(c) show that the Ge-O fraction after SHII with a fluence of $\sim 4.0 \times$ $10^{13} \mathrm{~cm}^{-2}$ is 13,33 , and $47 \%$, for the energies of 27,54 , and $185 \mathrm{MeV}$, respectively.

By adding the amorphous and Ge-O fractions together for each irradiation energy and fluence, we can plot the total fraction of Ge atoms in a noncrystalline environment. Such fraction is compared in Fig. 4 to calculations based on the overlap model, ${ }^{32}$ which considers that each ion creates a cylindrical damaged area around its path and amorphization is reached after a certain number of cylindrical regions overlap. 

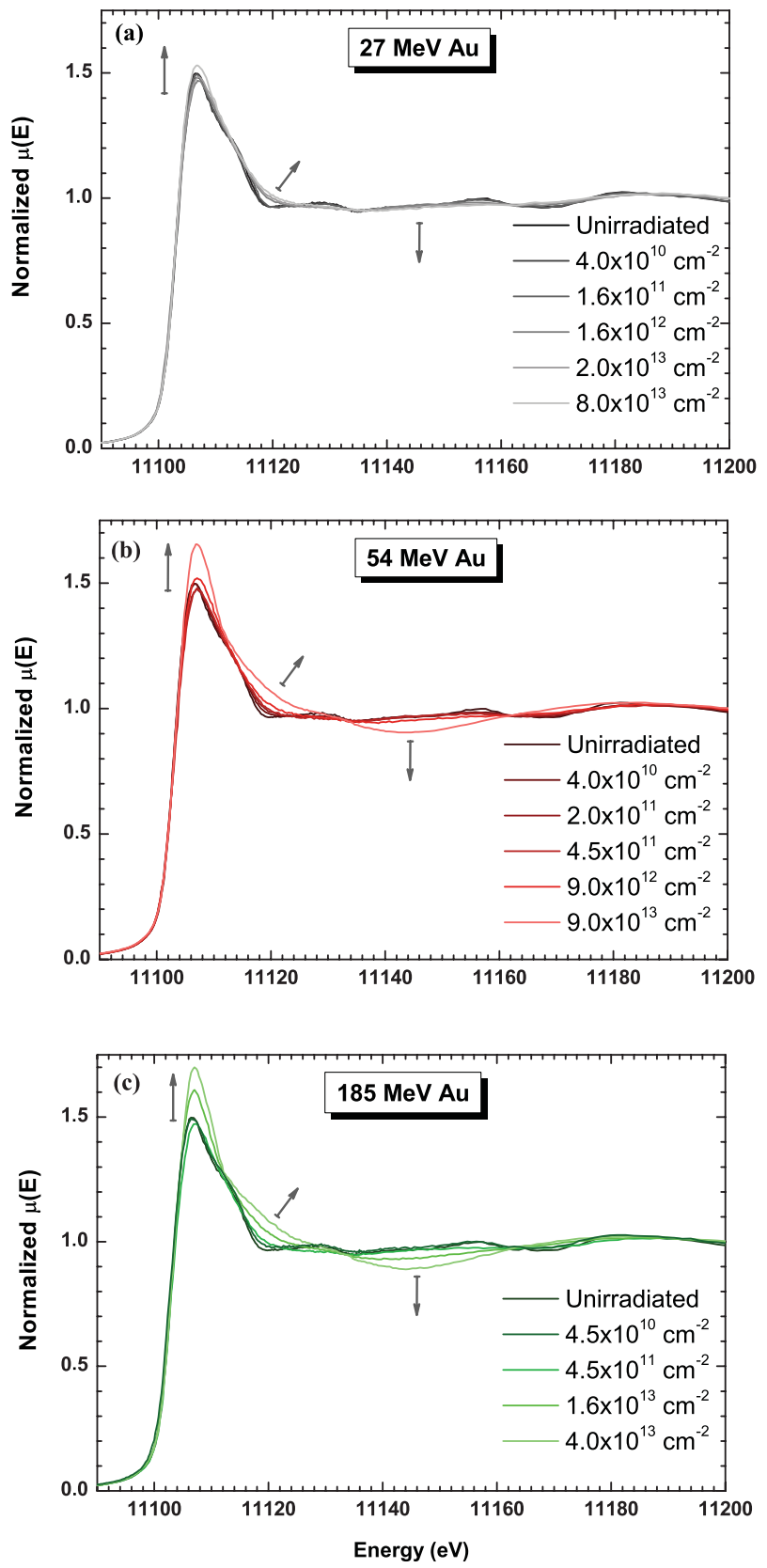

FIG. 2. (Color online) XANES spectra obtained for (a) $27 \mathrm{MeV}$, (b) $54 \mathrm{MeV}$, and (c) $185 \mathrm{MeV} \mathrm{Au}$ irradiation of Ge NPs samples at representative fluences, as listed on the legends. Lighter line traces represent higher fluences. The arrows indicate regions of the spectra that change progressively with irradiation fluence.

In principle, it could be assumed that the damaged area created by each ion is given by the ion track radius in $\mathrm{a}-\mathrm{SiO}_{2}$ for each SHII energy, but using such values yields poor agreement between experiment and calculations, as it is illustrated in Fig. 4 for $185 \mathrm{MeV}$ by the difference between experimental points (open diamond symbols) and the calculation with the radius of damaged region given by the ion track radius (dotted line). The best agreement between experiment (symbols) and calculation (full lines) is achieved when the radii of the damaged regions are taken as 3.0, 7.4, and $9.7 \mathrm{~nm}$ for the SHII energies of 27,54 , and $185 \mathrm{MeV}$, for which the ion track
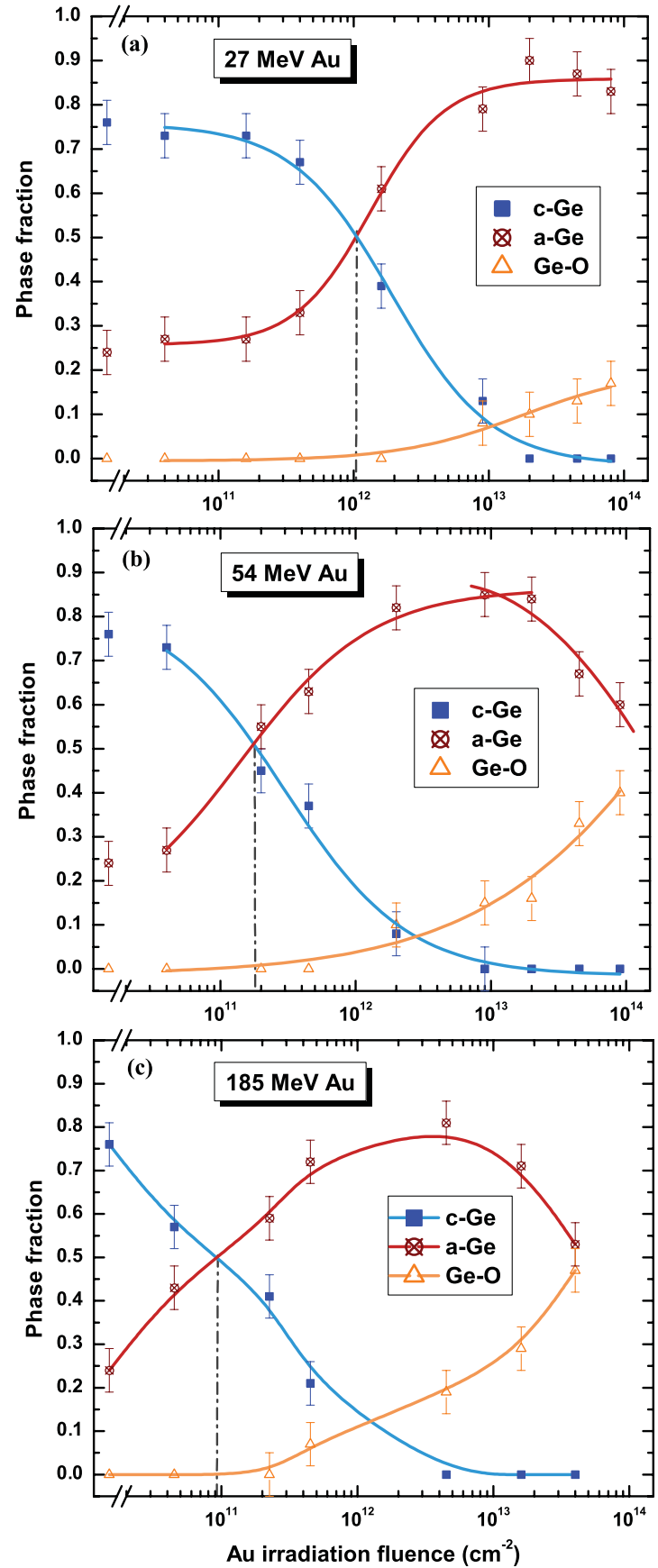

FIG. 3. (Color online) Fraction of Ge atoms in crystalline (c-Ge), amorphous (a-Ge), and oxidized (Ge-O) environments as a function of irradiation fluence for (a) $27 \mathrm{MeV}$, (b) $54 \mathrm{MeV}$, and (c) $185 \mathrm{MeV}$ $\mathrm{Au}$ irradiation. Symbols represent linear combination fit results from XANES data and solid lines are just guides to the eye. The vertical dash-dot lines indicate the fluence necessary to reduce the c-Ge fraction to $\sim 0.5$. Notice the break on the horizontal scale between unirradiated and irradiated points.

radii in $\mathrm{a}-\mathrm{SiO}_{2}$ are $2.5,4.0$, and $5.0 \mathrm{~nm}$, respectively. ${ }^{24}$ This can be understood considering that, for the formation of a track in $\mathrm{a}-\mathrm{SiO}_{2}$, the energy deposited by the ion must produce a local temperature increase above the $\mathrm{a}_{-} \mathrm{SiO}_{2}$ melting point (melting temperature $\left.T_{M} \sim 1970 \mathrm{~K}\right),{ }^{8}$ which is $\sim 63 \%$ higher than for bulk c-Ge $\left(T_{M}=1210 \mathrm{~K}\right)$ and also higher than for 




FIG. 4. (Color online) Total fraction of Ge atoms in a noncrystalline environment as a function of irradiation fluence for 27, 54, and $185 \mathrm{MeV} \mathrm{Au}$ irradiations. Symbols are experimental data, and solid lines are best fits with the overlap model. The dashed line represents overlap model results for a damage cross-section equivalent to the track diameter in $\mathrm{a}-\mathrm{SiO}_{2}$ under $185 \mathrm{MeV} \mathrm{Au}$ irradiation (compare to the experimental data for the same energy, plotted as open diamonds). Notice the break on the horizontal scale between unirradiated and irradiated points.

Ge NPs, even considering that embedded Ge NPs may melt at higher temperature relative to bulk Ge depending on their size $\left(T_{M} \sim 1400 \mathrm{~K}\right.$ for 5 -nm-diameter Ge NPs in $\left.\mathrm{a}-\mathrm{SiO}_{2}\right){ }^{33}$ Thus, electrons which are not hot enough to produce melting in a- $\mathrm{SiO}_{2}$ are still potentially hot enough to produce melting of the Ge NPs at distances from the ion trajectory larger than the track radius in a- $\mathrm{SiO}_{2}$. From this analysis, we can conclude that the amorphization of Ge NPs in the present case does not necessarily require the formation of ion tracks in the $\mathrm{a}-\mathrm{SiO}_{2}$ matrix, unlike the elongation process for embedded semiconductor and metal NPs.

In principle, the results from the overlap model could also be used to estimate the average number of ion impacts needed to amorphize a Ge NP, but several factors preclude a reliable estimate. First, the samples contain a size distribution of $\mathrm{Ge}$ $\mathrm{NPs}$, with diameters varying from 6 to $12 \mathrm{~nm}$. It has been shown that the smaller the Ge NPs are, the easier to amorphize them under irradiation at the nuclear energy deposition regime. ${ }^{30} \mathrm{~A}$ size-dependent trend is also expected here, since the energy per atom deposited on each Ge NP will be a function of the distance from the ion trajectory and of the particle size. Thus, it is likely that, at a given SHII energy, some Ge NPs are amorphized by the passage of a single ion, while other NPs require more ion impacts. Second, many ion impacts considered by the overlap model may not hit a single Ge NP, since the peak concentration of $\mathrm{Ge}$ in $\mathrm{a}-\mathrm{SiO}_{2}$ is 3.0 at.\% only. Quantitative estimation of the combined effect from such contributions is not trivial, so that no average number of impacts required for amorphization can be reported here.
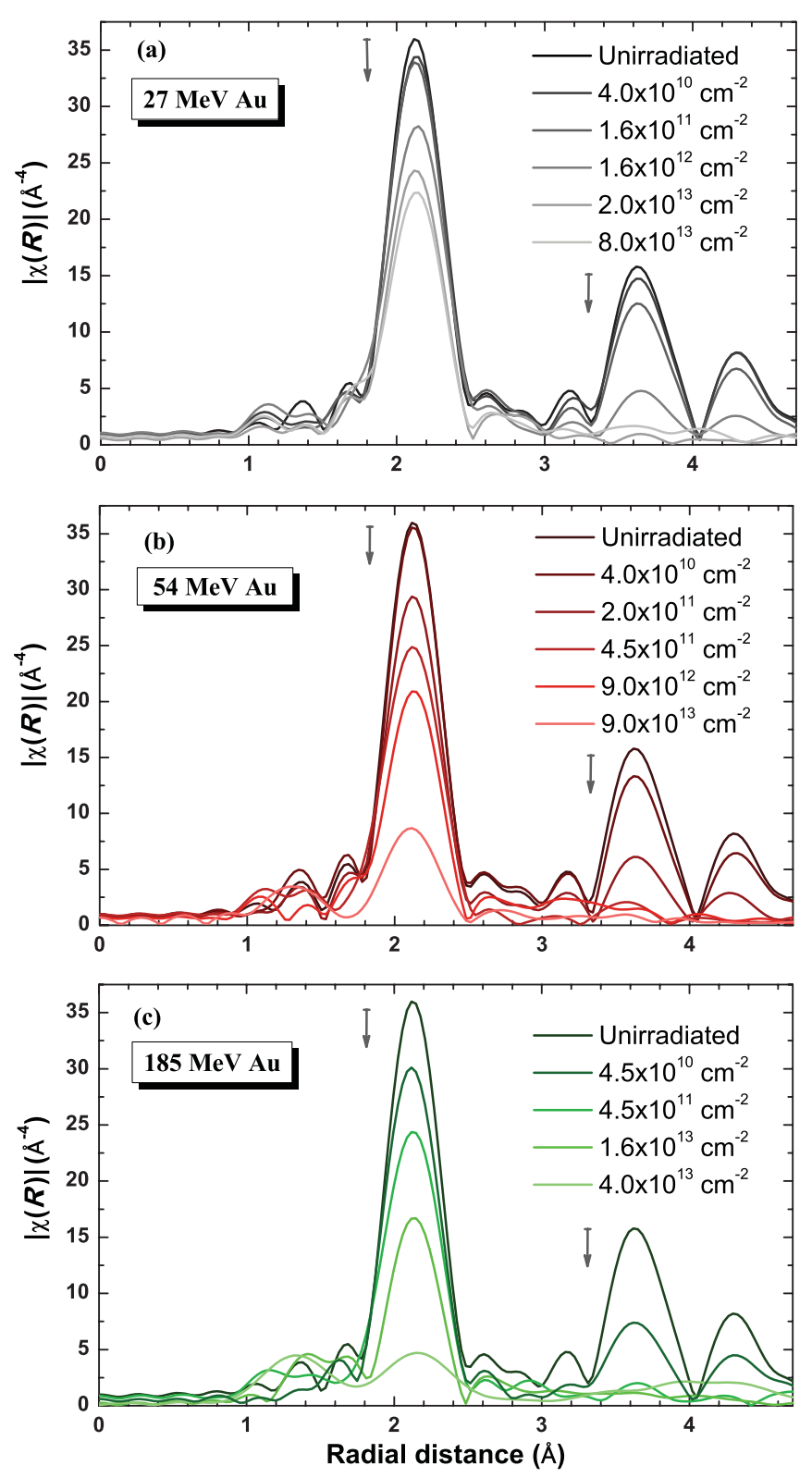

FIG. 5. (Color online) Nonphase-corrected EXAFS spectra, plotted in radial distance $(R)$ space, obtained after (a) $27 \mathrm{MeV}$, (b) $54 \mathrm{MeV}$, and (c) $185 \mathrm{MeV} \mathrm{Au}$ irradiation of Ge NPs samples at representative fluences, as listed on the legends. Lighter line traces represent higher fluences. The arrows indicate regions of the spectra that change progressively with irradiation fluence.

\section{B. EXAFS results}

Figures 5(a)-5(c) show Fourier-transformed (FT) EXAFS spectra (nonphase-corrected) corresponding to the same samples shown in Figs. 2(a)-2(c). Changes in nearest neighbor (NN) peak amplitudes are readily apparent, again evolving more rapidly with fluence for the higher irradiation energies. The accentuated decrease in amplitude for the second and third peaks in the FT spectra, followed by their disappearance at intermediate irradiation fluences (the higher the energy, the lower the fluence), is a clear indication that the Ge NPs are being amorphized. The second and third peaks in the FT contain the contributions from second and third Ge NN shells 
plus multiple scattering signals involving atoms in the first and second NN shells. Here, the vanishing of such contributions means loss of medium-range order, as previously demonstrated for bulk Ge. ${ }^{34}$ On the other hand, the first peak in the FT spectra corresponds to the signal from the first Ge NN shell only. The decrease in amplitude and broadening of the first peak can be ascribed to an increase in structural disorder (increase in the variance of the interatomic distance distribution) and/or to a decrease in average coordination number (decrease in the average number of $\mathrm{Ge}-\mathrm{Ge}$ bonds due to formation of $\mathrm{Ge}-\mathrm{O}$ bonds or to a decrease in Ge NP size). The XANES and TEM results presented here clearly demonstrate that, at low irradiation fluences, the increase in structural disorder is the main factor responsible for the decrease in first NN peak amplitudes, while at higher irradiation fluences, the decrease in $\mathrm{Ge}-\mathrm{Ge}$ coordination number becomes more pronounced. This is further confirmed by the structural parameters obtained by fitting the EXAFS data shown in Fig. 5 according to the procedure described in Sec. II.

Structural parameters for the first Ge NN shell-namely the average number of neighboring atoms (coordination number), mean interatomic distance, variance of the interatomic distance distribution (mean-square relative displacement or MSRD), and asymmetry of the interatomic distance distribution (third cumulant) - are shown in Figs. 6(a)-6(c) as a function of irradiation fluence for SHII at 27, 54, and $185 \mathrm{MeV}$. Again, the more pronounced changes are observed for the $185 \mathrm{MeV}$ case, reinforcing that the features observed in this work are a result of electronic energy loss rather than ballistic collisions.

Figure 6(a) shows the evolution of the coordination numbers with irradiation fluence for samples under 27, 54, and $185 \mathrm{MeV}$ SHII (down triangles, crossed circles, and open diamonds, respectively). The coordination numbers decrease with irradiation fluence for all energies (as highlighted by the dotted lines, plotted to guide the eye), as expected from the decrease in peak amplitudes mentioned above, but part of the decrease in amplitude for the first Ge-Ge peak, for higher irradiation fluences, comes from the presence of a significant fraction of $\mathrm{Ge}-\mathrm{O}$ bonds in the total EXAFS signal. This will affect the values for the Ge-Ge coordination numbers, which will appear smaller than the true value. To account for this effect, coordination numbers were corrected using the oxide fractions determined by XANES for SHII at $4.5 \times 10^{13}$ and $9.0 \times 10^{13} \mathrm{~cm}^{-2}$ under $54 \mathrm{MeV}$ (plotted as stars), as well as SHII at $4.5 \times 10^{12}$ and $1.6 \times$ $10^{13} \mathrm{~cm}^{-2}$ under $185 \mathrm{MeV}$ (plotted as hexagons), as an example. The corrected coordination numbers indicate a decrease to an average number of three nearest Ge neighbors, which can be ascribed to a combination of factors: decrease in coordination due to a decrease in NP size, further structural modification in the NPs (as discussed below), and/or the combined effect of fourfold coordinated atoms in the Ge NPs and Ge dimers and trimers dissolved in the matrix.

The MSRD, which can be regarded as a measure of the total disorder or the variance of the interatomic distance distribution, is plotted as a function of irradiation fluence in Fig. 6(b). For the Ge NPs irradiated with $27 \mathrm{MeV} \mathrm{Au}$, the MSRD increases with irradiation fluence up to a value of $\sim 4.2 \times 10^{-3} \AA^{2}$ (corresponding to the MSRD value measured for bulk a-Ge, as indicated by the full line in the figure) and then


FIG. 6. (Color online) Structural parameters for the first GeGe nearest neighbor shell as obtained from the EXAFS data analysis for samples irradiated with 27,54 , and $185 \mathrm{MeV}$ : (a) coordination numbers, (b) mean-square relative displacements (MSRDs), (c) relative interatomic distances, and (d) third cumulants. Symbols are experimental results, and dotted lines are guides to the eyes. The full line in panel (b) indicates the MSRD value for bulk a-Ge. The symbols corresponding to energies labeled with asterisks in panel (a) are the values of the coordination numbers corrected by the oxide fraction at the given irradiation fluences.

remains approximately constant. Notice the XANES results do not indicate significant Ge NP dissolution for SHII with $27 \mathrm{MeV}$. As discussed in previous works, this indicates the NPs are rendered amorphous by the ion beam. ${ }^{30} \mathrm{~A}$ similar trend is initially observed for the Ge NPs irradiated with 54 and $185 \mathrm{MeV} \mathrm{Au}$, with the MSRD reaching the a-Ge value at lower irradiation fluences as the irradiation energy increases. Again, this indicates that amorphization of the Ge NPs in this study is a result of electronic excitations, but 
unlike for the $27 \mathrm{MeV}$ case, the MSRD increases past the a-Ge value for irradiation with 54 and $185 \mathrm{MeV}$ at higher fluences, the effect being more pronounced for the highest energy. Such behavior is most likely the combined effect of an increase in the variance of interatomic distances due to the coexistence of Ge NPs and Ge dimers and trimers dispersed in the matrix-since NP dissolution is more pronounced for the highest irradiation energies-and of further structural modifications in the Ge NPs. It has been shown that SHII of bulk a-Ge layers with $185 \mathrm{MeV}$ Au ions leads to a volume expansion due to void formation and the transformation to a spongelike porous structure at high fluences. ${ }^{35}$ Our EXAFS results for the highest irradiation energies suggest Ge NPs also transform progressively into a porous structure, concomitantly with the shape-change process. This is in agreement with the high-resolution TEM analysis from Ref. 11, which also suggests the formation of a low-density spongelike phase in Ge NPs under SHII with $38 \mathrm{MeV}$ I ions. We note that, for all cases, the Ge NPs are first amorphized and then evolve towards a porous structure.

Figure 6(c) presents the relative mean interatomic distance (mean interatomic distance in the Ge NPs minus mean interatomic distance in bulk c-Ge) obtained for the Ge NPs under all SHII conditions. For the Ge NPs irradiated with $27 \mathrm{MeV} \mathrm{Au}$, the interatomic distance increases with irradiation fluence up to approximately the value corresponding to bulk $\mathrm{a}-\mathrm{Ge}$ and then remains constant (as happens for the MSRD). Again, this is a clear indication of Ge NP amorphization by SHII. For the Ge NPs irradiated with $54 \mathrm{MeV} \mathrm{Au}$, the interatomic distance also increases with irradiation fluence, reaching the a-Ge value at a lower fluence compared to the $27 \mathrm{MeV}$ case, but even though the MSRD for SHII with 54 $\mathrm{MeV}$ increases above the bulk a-Ge value, the interatomic distance remains around the bulk a-Ge value. This can be understood as resulting from equivalent contributions from NP dissolution (driving the interatomic distance to lower values through the generation of $\mathrm{Ge}$ dimers/trimers in the matrix) and of further structural changes in the Ge NPs (driving the interatomic distance to higher values through the formation of a porouslike structure). This way, the mean value of the interatomic distance may remain approximately constant even though the variance is increasing. On the other hand, for SHII with $185 \mathrm{MeV}$, the Ge-Ge interatomic distance in the samples starts to rise towards the bulk a-Ge value at lower irradiation fluences, but then drops below the bulk c-Ge interatomic distance as the irradiation fluence is increased. This could result from more pronounced Ge NP dissolution at $185 \mathrm{MeV}$, in agreement with XANES and TEM observations.

\section{TEM results}

The structural evolution of the Ge NPs under SHII reported in the previous subsections can be correlated with their morphological evolution through TEM analysis. Figures 7(a)7(1) show representative images of Ge NP samples obtained for selected fluences after SHII with $27 \mathrm{MeV}$ Au (left column), $54 \mathrm{MeV} \mathrm{Au}$ (middle column), and $185 \mathrm{MeV} \mathrm{Au}$ (right column).

For SHII at $27 \mathrm{MeV}$, Fig. 7(a) shows Ge NPs remain spherical after irradiation with $1.6 \times 10^{12} \mathrm{~cm}^{-2}$, with the same average mean size as the unirradiated Ge NPs (not


FIG. 7. (Color online) TEM images obtained from samples irradiated with: $27 \mathrm{MeV} \mathrm{Au}$ to a fluence of (a) $1.6 \times 10^{12}$, (b) $9.0 \times 10^{12}$, (c) $2.0 \times 10^{13}$, and (d) $8.0 \times 10^{13} \mathrm{~cm}^{-2} ; 54 \mathrm{MeV} \mathrm{Au}$ to a fluence of (e) $2.0 \times 10^{11}$, (f) $2.0 \times 10^{12}$, (g) $2.0 \times 10^{13}$, and (h) $4.5 \times 10^{13} \mathrm{~cm}^{-2} ; 185 \mathrm{MeV}$ Au to a fluence of (i) $4.5 \times 10^{11}$, (j) $4.5 \times 10^{12}$, (k) $1.6 \times 10^{13}$, and (l) $4.0 \times 10^{13} \mathrm{~cm}^{-2}$. The direction of the ion beam was from top to bottom in all cases, as indicated by the arrow in frame (j). Full circles highlight NPs elongated along the beam direction, while dashed circles highlight NPs elongated perpendicular to the beam.

shown). According to the XANES and EXAFS results, at such fluence the c-Ge fraction in the Ge NP ensemble has already decreased to less than $50 \%$, indicating amorphization does not necessarily involve changes in NP shape and/or size. This is further confirmed in Fig. 7(b), corresponding to the Ge NP sample irradiated with $9.0 \times 10^{12} \mathrm{~cm}^{-2}$. At this irradiation fluence, the XANES and EXAFS results indicate a c-Ge fraction of about $10 \%$ and, again, TEM shows spherical particles with the same average mean size as the unirradiated sample. Morphological changes in the Ge NPs start becoming apparent at irradiation fluences where no c-Ge fraction remains, as illustrated in Fig. 7(c) for irradiation with a fluence of $2.0 \times 10^{13} \mathrm{~cm}^{-2}$. The biggest Ge NPs still remain spherical, while the smaller NPs become prolate spheroids with the major diameter aligned with the beam direction [indicated by the arrow in Fig. 7(j)]. Note that, at this fluence, a small fraction of $\mathrm{Ge}-\mathrm{O}$ bonds is formed according to the XANES results, indicating the shape-change process is concurrent with some dispersion of Ge into the 
a- $\mathrm{SiO}_{2}$ matrix and/or intermixing at the $\mathrm{NP} /$ matrix interface, which do not take place during the amorphization process. As will be discussed later, the amorphization of the Ge NPs depends solely on the energy deposited into the NPs, while the elongation process depends on the energy deposited on both the Ge NP and a-SiO $\mathrm{Sitrix}_{2}$ (formation of a molten track). Figure 7(d) depicts a very interesting scenario for the Ge NPs irradiated with $8.0 \times 10^{13} \mathrm{~cm}^{-2}$. At this irradiation fluence, the vast majority of NPs shows some change in shape, but not always in the same direction. While the smaller Ge NPs become prolate spheroids or rods elongated parallel to the ion beam direction (NPs highlighted with a full circle), the bigger Ge NPs become oblate spheroids elongated perpendicular to the ion beam direction (NPs highlighted with a dashed circle). Such behavior is consistent with that previously reported in Refs. 11 and 12 for the SHII with $38 \mathrm{MeV}$ I beams of Ge NPs embedded in a-SiO $\mathrm{Si}_{2}$ with mean diameters of $8,18,26$, and $37 \mathrm{~nm}$; although the Ge NP sizes (the biggest Ge NPs in this work correspond to the smallest Ge NPs in the previous reports) and the irradiation conditions (beam energy, fluence, energy loss) are different in absolute values, their relative variation is somewhat similar for this case and the previous works. ${ }^{11,12}$ Recalling the data presented in Fig. 1, note that for SHII with $27 \mathrm{MeV} \mathrm{Au}$, almost all Ge NPs in the sample range from slightly bigger to much bigger than the mean track diameter in $\mathrm{a}-\mathrm{SiO}_{2}$. This will be further discussed in the next subsection.

The TEM images for SHII with $54 \mathrm{MeV}$ show some similarities and some differences with respect to the $27 \mathrm{MeV}$ case. Figures 7(e) and 7(f) present spherical Ge NPs of the same average size after irradiation with fluences of $2.0 \times$ $10^{11}$ and $2.0 \times 10^{12} \mathrm{~cm}^{-2}$, respectively. According to the XANES/EXAFS results, the c-Ge fraction in such samples is $45 \%$ and $5 \%$, respectively, indicating that amorphization of the Ge NPs proceeds without significant changes in shape and size, as for the $27 \mathrm{MeV}$ case. It is important to stress, though, that the fluences for amorphization at $54 \mathrm{MeV}$ SHII [Figs. 7(e) and 7(f)] are approximately one order of magnitude lower than at $27 \mathrm{MeV}$ SHII [Figs. 7(a) and 7(b)], demonstrating faster amorphization with higher electronic energy deposition. Shape transformation becomes observable after irradiation with approximately $2.0 \times 10^{13} \mathrm{~cm}^{-2}$, as illustrated in Fig. $7(\mathrm{~g})$. Similar to the $27 \mathrm{MeV}$ regime, the shape changes only become observable after the Ge NPs are amorphized and occur concurrently with the appearance of a fraction of $\mathrm{Ge}-\mathrm{O}$ bonds. Nevertheless, unlike the $27 \mathrm{MeV}$ case, where smaller Ge NPs start elongating first, both bigger and smaller Ge NPs begin to change shape at the intermediate fluence depicted in Fig. 7(g) under $54 \mathrm{MeV}$ SHII. A more striking difference relative to the $27 \mathrm{MeV}$ case is depicted in Fig. 7(h): after irradiation at $54 \mathrm{MeV}$ with $4.5 \times 10^{13} \mathrm{~cm}^{-2}$, all Ge NPs become prolate spheroids/rods elongated parallel to the ion beam direction. No perpendicular elongation is observed at this energy, unlike for the $27 \mathrm{MeV} \mathrm{Au}$ regime and the previous works mentioned above. ${ }^{11,12}$ Referring to Fig. 1 once more, it can be seen that, for SHII with $54 \mathrm{MeV} \mathrm{Au}$, about $28 \%$ of the Ge NPs are now smaller than the average track diameter in $\mathrm{a}-\mathrm{SiO}_{2}$, while the difference between the biggest NP diameter and the track diameter is not as pronounced as at the lower energy regime. Furthermore, the probability of track overlaps is higher for 54
$\mathrm{MeV}$ than $27 \mathrm{MeV}$ for any given irradiation fluence. These effects will be further discussed in the next subsection.

Figures 7(i)-7(1) summarize the TEM results for Ge NPs under SHII with $185 \mathrm{MeV} \mathrm{Au}$ beams. For an irradiation fluence of $4.5 \times 10^{11} \mathrm{~cm}^{-2}$, the c-Ge fraction already drops to $20 \%$, while the Ge NPs remain spherical, as shown in Fig. 7(i). As for 27 and $54 \mathrm{MeV}$, amorphization of Ge NPs under SHII with $185 \mathrm{MeV} \mathrm{Au} \mathrm{proceeds} \mathrm{without} \mathrm{significant}$ changes in NP shape and size, but is completed at even lower irradiation fluences. Figure 7(j) shows Ge NPs after irradiation with $4.5 \times 10^{12} \mathrm{~cm}^{-2}$, which are mostly spherical, although some deformation is apparent in a few NPs. For such irradiation fluence, the c-Ge fraction is null, and the fraction of $\mathrm{Ge}-\mathrm{O}$ bonds is about $20 \%$, indicating that, for the $185 \mathrm{MeV}$ irradiation, the elongation process starts at lower fluences compared to the other SHII energies. Also, the fluence interval between complete amorphization of the Ge NPs and the onset of NP deformation is smaller, possibly indicating that some amorphized Ge NPs may start to change shape while other NPs are still being amorphized. Complete Ge NP elongation seems to be reached at fluences around $1.6 \times 10^{13}$ $\mathrm{cm}^{-2}$, as illustrated in Fig. 7(k). Similar to the $54 \mathrm{MeV}$ case, only elongation parallel to the ion beam direction is observed. Figure 1 indicates that, for $185 \mathrm{MeV}$ irradiation, $83 \%$ of the Ge NPs are smaller than the ion track diameter, while no NP exceeds the ion track in size by more than a track radius. This will be further discussed below. Finally, Fig. 7(1) shows that further irradiation to a fluence of $4.0 \times 10^{13} \mathrm{~cm}^{-2}$ causes the elongated Ge NPs to start dissolving, and a considerable amount of Ge becomes dispersed in the matrix, as consistent with a Ge-O fraction of $45 \%$ determined by XANES.

\section{DISCUSSION}

The results presented here indicate the SHII of Ge NPs embedded in $\mathrm{a}-\mathrm{SiO}_{2}$ leads to four main effects: amorphization, change in shape, further structural modification (possibly formation of a porous-like structure), and dissolution.

The amorphization of the Ge NPs precedes the other effects and may be a necessary condition for their manifestation. Electronic excitations and ionization can produce substantial atomic rearrangements ${ }^{36}$ in insulators and amorphous solids, but most crystalline materials are not structurally perturbed by SHII. For example, local melting and amorphization in c-Ge (and c-Si) cannot be achieved with SHII for single ions with electronic energy loss $S_{e}$ below $\approx 35 \mathrm{keVnm}^{-1}$, indicative of a significant difference in electron-phonon coupling between the amorphous and crystalline phases. ${ }^{35}$ Amorphization of bulk c-Ge due to $S_{e}$ was observed only for irradiation with $\mathrm{C}_{60}$ (fullerene) clusters of initial energy $20-40 \mathrm{MeV},{ }^{37}$ where the deposited energy density is higher than for elemental ion beams.

Nevertheless, the initially crystalline Ge NPs in this study were easily rendered amorphous by SHII with Au beams, at energies where no changes are observed for bulk c-Ge. Previous studies indicate that the existence of point defects and clusters of point defects increases the electron-phonon coupling efficiency in semiconductors irradiated with swift heavy ions. ${ }^{38}$ Thus, electron-phonon coupling should be more efficient for the Ge NPs than bulk c-Ge since, even in the 
as-formed state, the NPs have disordered surfaces/interface with the matrix, resulting in an amorphouslike outer shell. ${ }^{22}$ Furthermore, explicit calculations show how an increase of the electron-phonon coupling constant (which is also attributed to defects) may increase the radius of a molten track. ${ }^{39}$ Consequently, the minimum energy deposited by electronic energy loss for melting should be lower for the NPs in relation to bulk. It is also plausible that the NP-matrix interface could hinder energy/heat dissipation compared to bulk Ge, thus better containing the energy inside the NP volume. The observed amorphization of Ge NPs potentially proceeds via a melt-and-quench process, since it is more pronounced for higher irradiation energies, revealing $S_{e}$ is the dominant effect. Thus, while the amorphization of Ge NPs at the nuclear energy loss $\left(S_{n}\right)$ irradiation regime proceeds via creation and accumulation of defects by collision cascades, characterizing a solid-solid transformation, ${ }^{30}$ the amorphization of Ge NPs by SHII may well comprise a solid-liquid-solid transformation. Given the low fluence ranges where the amorphization of $\mathrm{Ge}$ NPs is observed, and based on the comparison of XANES data and the overlap model, it is very likely that the amorphization of Ge NPs does not necessitate the formation of a molten track in the a- $\mathrm{SiO}_{2}$ matrix directly surrounding the NPs, but only a certain amount of deposited energy per atom into the NPs due to the passage of an ion in close proximity.

The shape-change process for embedded Ge NPs, on the other hand, is dependent on the presence of an ion track in the a- $\mathrm{SiO}_{2}$ matrix, allowing for the flow of molten NP and matrix material, which is constricted by the ion track diameter. The TEM images presented in Fig. 7 clearly show that NP elongation proceeds gradually with irradiation fluence, involving many ion impacts until complete deformation of the Ge NPs is achieved.

A relationship to estimate the largest diameter for an embedded Ge NP to be molten due to a given $S_{e}$ deposited by SHII was proposed in Ref. 12. Applying such a relationship to SHI Au beams of initial energy 27, 54, and $185 \mathrm{MeV}$ results in maximum Ge NP diameters for melting of about 23, 33, and $55 \mathrm{~nm}$. This indicates all direct ion impacts should cause melting at the irradiation conditions used in this work, since the biggest Ge NPs have a diameter of about $16 \mathrm{~nm}$. This is consistent with Ge NP elongation being observed in all cases.

As for the elongation along different directions, Ref. 12 proposes a model where elongation perpendicular to the ion beam direction is explained by the higher density of liquid Ge compared to solid c-Ge (5\% difference) and the Ge NPs being bigger than the track diameter in the $\mathrm{a}-\mathrm{SiO}_{2}$ matrix. Basically, if only one region of the particle were hit by the ion track, then material flow would occur only within that region surrounded by the molten track, even though energy would be distributed within the whole Ge NP, because the matrix around the rest of the NP would still be solid, constricting material flow. Within the track diameter, the interfaces between molten matrix and NP would move into the Ge NP to allow for the increase in density when Ge becomes liquid, thereby reducing the tensile stress built up during the short melting period (10 to $100 \mathrm{ps}$ ), where the Ge NP would necessarily reduce in volume. Within a few tens of picoseconds, the molten Ge NP would then cool, reaching (due to the extreme cooling rate) a strongly undercooled liquid state. From that state, it would resolidify suddenly without a chance of re-expansion in the track, which would now be solid.

Such a model can explain what is observed in the present case for Ge NPs of diameter $D_{N P} \sim 10 \mathrm{~nm}$ or more under SHII with $27 \mathrm{MeV}$ Au (5-nm track diameter $D_{T}$ ). But it fails to explain what is observed for the other Ge NPs $\left(\sim 7<D_{N P}<\right.$ $10 \mathrm{~nm}$ ) which are still bigger than the track diameter but elongate parallel to the beam direction. The same disagreement is observed for SHII at $54 \mathrm{MeV}\left(D_{T}=8 \mathrm{~nm}\right)$, where all Ge NPs $\left(D_{N P} \leqslant 16 \mathrm{~nm}\right)$ elongate parallel to the ion beam direction. Although the data presented here confirms the direction of elongation depends on both particle size and track diameter, it suggests that parallel elongation is not limited to the case $D_{N P} \leqslant D_{T}$, but is also observed for $D_{N P}$ as big as $2 \cdot D_{T}$.

On the other hand, if the Ge NP diameter is smaller or roughly the same size as the ion track, elongation parallel to the ion beam direction is intuitively understood as due to material flow within the molten track. The results for SHII with $185 \mathrm{MeV} \mathrm{Au}$ ions are thus those that could be expected for the given irradiation conditions.

Transmission electron microscopy results in previous works $^{11,12}$ gave qualitative indication that the interaction of the molten tracks with the Ge NPs resulted in dissolution of some $\mathrm{Ge}$ atoms in the $\mathrm{a}-\mathrm{SiO}_{2}$ matrix at high irradiation fluences ( 1 to $4 \times 10^{15} \mathrm{~cm}^{-2}$ ), forming small spherical satellites (composed of both Ge and $\mathrm{O}$ atoms) around the elongated Ge NPs. The results presented here allowed quantification of the fraction of $\mathrm{Ge}$ atoms binding to $\mathrm{O}$ as a function of SHII fluence and energy, indicating that the formation of $\mathrm{Ge}-\mathrm{O}$ bonds starts at the early stages of the elongation process. This demonstrates that dissolution of the Ge NPs occurs concurrently with (and not exclusively after) NP elongation, becoming more pronounced as the irradiation fluence increases.

The evolution of the mean coordination number, mean value, and variance of the interatomic distance distribution for the first Ge NN shell revealed the structural evolution of the Ge NPs initially involves a transition from a crystalline state to an amorphous state, followed by a further transition into a state of higher structural disorder, possibly porouslike Ge, as suggested in Ref. 11, and Ge NP dissolution (with the formation of very small $\mathrm{Ge}$ clusters and $\mathrm{Ge}-\mathrm{O}$ bonds).

\section{CONCLUSIONS}

It was shown that crystalline Ge NPs are easily amorphized by SHII, while bulk c-Ge is not. The amorphization occurs at lower fluences for the highest SHII energy (highest $S_{e}$ ), indicating that electronic energy loss is the dominant process.

Ge NP elongation was observed at fluences higher than those required for amorphization, with a combination of initial NP size and SHII $S_{e}$ determining the fluence required for shape change and the direction of elongation. Both parallel and perpendicular elongations with respect to the ion beam direction were observed as a function of Ge NP diameter and irradiation conditions. Perpendicular elongation was only observed for cases where the Ge NP diameter was approximately equal to or bigger than twice the track diameter in $\mathrm{a}-\mathrm{SiO}_{2}$, somewhat in contradiction to previous models. Further investigation is needed to better explain this behavior peculiar to embedded Ge NPs. 
The formation of Ge-O bonds due to NP dissolution and/or intermixing with the a-SiO $\mathrm{S}_{2}$ matrix was observed to start at the early stages of the shape-change process and increases significantly with irradiation fluence, the effect being more pronounced for higher irradiation energies. This indicates the shaping of embedded Ge nanostructures by SHII will always result in some loss of Ge from the NPs.

In principle, it would be possible to recover the crystalline state by annealing the ion-beam modified nanostructures. However, the process may lead to undesirable changes, such as a return to spherical NP shape and an increase in NP size. This indicates the ion beam engineering of semiconductor nanocrystalline structures must be considered very carefully.

\section{ACKNOWLEDGMENTS}

The authors acknowledge the ARC for financial support. We thank the 14UD accelerator technical team for the assistance during the irradiations.
*Email address: leandro.langie@ufrgs.br

${ }^{1}$ S. Klaumünzer, Nucl. Instrum. Methods Phys. Res., Sect. B 244, 1 (2006).

${ }^{2}$ K. H. Heinig, T. Muller, B. Schmidt, M. Strobel, and W. Moller, Appl. Phys. A: Mater. Sci. Process. 77, 17 (2003).

${ }^{3}$ T. van Dillen, A. van Blaaderen, and A. Polman, Mater. Today 7 , 40 (2004).

${ }^{4}$ G. Rizza, E. A. Dawi, A. M. Vredenberg, and I. Monnet, Appl. Phys. Lett. 95, 043105 (2009).

${ }^{5}$ C. D’Orlèans, J. P. Stoquert, C. Estournès, C. Cerruti, J. J. Grob, J. L. Guille, F. Haas, D. Muller, and M. Richard-Plouet, Phys. Rev. B 67, 220101 (2003).

${ }^{6}$ M. C. Ridgway, P. Kluth, R. Giulian, D. J. Sprouster, L. L. Araujo, C. S. Schnohr, D. J. Llewellyn, A. P. Byrne, G. J. Foran, and D. J. Cookson, Nucl. Instrum. Methods Phys. Res., Sect. B 267, 931 (2009).

${ }^{7}$ A. Oliver, J. A. Reyes-Esqueda, J. C. Cheang-Wong, C. E. Román-Velázquez, A. Crespo-Sosa, L. Rodríguez-Fernández, J. A. Seman, and C. Noguez, Phys. Rev. B 74, 245425 (2006).

${ }^{8}$ K. Awazu, X. Wang, M. Fujimaki, J. Tominaga, H. Aiba, Y. Ohki, and T. Komatsubara, Phys. Rev. B 78, 054102 (2008).

${ }^{9}$ Y. K. Mishra, F. Singh, D. K. Avasthi, J. C. Pivin, D. Malinovska, and E. Pippel, Appl. Phys. Lett. 91, 063103 (2007).

${ }^{10}$ R. Giulian, P. Kluth, L. L. Araujo, D. J. Sprouster, A. P. Byrne, D. J. Cookson, and M. C. Ridgway, Phys. Rev. B 78, 125413 (2008).

${ }^{11}$ B. Schmidt, A. Mucklich, L. Rontzsch, and K. H. Heinig, Nucl. Instrum. Methods Phys. Res., Sect. B 257, 30 (2007).

${ }^{12}$ B. Schmidt, K. H. Heinig, A. Mücklich, and C. Akhmadaliev, Nucl. Instrum. Methods Phys. Res., Sect. B 267, 1345 (2009).

${ }^{13}$ D. J. Sprouster, R. Giulian, C. S. Schnohr, L. L. Araujo, P. Kluth, A. P. Byrne, G. J. Foran, B. Johannessen, and M. C. Ridgway, Phys. Rev. B 80, 115438 (2009).

${ }^{14}$ S. Roorda, T. van Dillen, A. Polman, C. Graf, A. van Blaaderen, and B. J. Kooi, Adv. Mater. 16, 235 (2004).

${ }^{15}$ M. C. Ridgway, R. Giulian, D. J. Sprouster, P. Kluth, L. L. Araujo, D. J. Llewellyn, A. P. Byrne, F. Kremer, P. F. P. Fichtner, G. Rizza, H. Amekura, and M. Toulemonde, Phys. Rev. Lett. 106, 095505 (2011)

${ }^{16}$ D. J. Sprouster, R. Giulian, L. L. Araujo, P. Kluth, B. Johannessen, D. J. Cookson, and M. C. Ridgway, J. Appl. Phys. 109, 113504 (2011).
${ }^{17}$ E. A. Dawi, G. Rizza, M. P. Mink, A. M. Vredenberg, and F. H. P. M. Habraken, J. Appl. Phys. 105, 074305 (2009).

${ }^{18}$ K. Awazu, X. Wang, T. Komatsubara, J. Watanabe, Y. Matsumoto, S. I. Warisawa, and S. Ishihara, Nanotechnology 20, 325303 (2009).

${ }^{19}$ G. Rizza, A. Dunlop, and A. Dezellus, Nucl. Instrum. Methods Phys. Res., Sect. B 256, 219 (2007).

${ }^{20}$ R. Giulian, F. Kremer, L. L. Araujo, D. J. Sprouster, P. Kluth, P. F. P. Fichtner, A. P. Byrne, and M. C. Ridgway, Phys. Rev. B 82, 113410 (2010).

${ }^{21}$ T. van Dillen, M. J. A. de Dood, J. J. Penninkhof, A. Polman, S. Roorda, and A. M. Vredenberg, Appl. Phys. Lett. 84, 3591 (2004).

${ }^{22}$ L. L. Araujo, R. Giulian, D. J. Sprouster, C. S. Schnohr, D. J. Llewellyn, P. Kluth, D. J. Cookson, G. J. Foran, and M. C. Ridgway, Phys. Rev. B 78, 094112 (2008).

${ }^{23}$ J. F. Ziegler, J. P. Biersack, and M. D. Ziegler, SRIM-The Stopping and Range of Ions in Matter (Lulu Press Co., Morrisville, 2008).

${ }^{24}$ P. Kluth, C. S. Schnohr, O. H. Pakarinen, F. Djurabekova, D. J. Sprouster, R. Giulian, M. C. Ridgway, A. P. Byrne, C. Trautmann, D. J. Cookson, K. Nordlund, and M. Toulemonde, Phys. Rev. Lett. 101, 175503 (2008).

${ }^{25}$ J. Hester, Average v.2.0.6, 2007. Available at [http://anbf2.kek.jp/ xafs-downloads.html].

${ }^{26}$ B. Ravel and M. Newville, J. Synchrotron Radiat. 12, 537 (2005).

${ }^{27}$ M. Newville, J. Synchrotron Radiat. 8, 322 (2001).

${ }^{28}$ S. D. Kelly and B. Ravel, AIP Conf. Proc. 882, 132 (2007).

${ }^{29}$ J. J. Rehr and R. C. Albers, Rev. Mod. Phys. 72, 621 (2000).

${ }^{30}$ M. Backman, F. Djurabekova, O. H. Pakarinen, K. Nordlund, L. L. Araujo, and M. C. Ridgway, Phys. Rev. B 80, 144109 (2009).

${ }^{31}$ M. C. Ridgway, G. de M. Azevedo, R. G. Elliman, C. J. Glover, D. J. Llewellyn, R. Miller, W. Wesch, G. J. Foran, J. Hansen, and A. Nylandsted-Larsen, Phys. Rev. B 71, 094107 (2005).

${ }^{32}$ J. F. Gibbons, Proc. IEEE 60, 1062 (1972).

${ }^{33}$ Q. Xu, I. D. Sharp, C. W. Yuan, D. O. Yi, C. Y. Liao, A. M. Glaeser, A. M. Minor, J. W. Beeman, M. C. Ridgway, P. Kluth, J. W. Ager, D. C. Chrzan, and E. E. Haller, Phys. Rev. Lett. 97, 155701 (2006).

${ }^{34}$ C. J. Glover, M. C. Ridgway, A. P. Byrne, K. M. Yu, G. J. Foran, C. Clerc, J. L. Hansen, and A. Nylandsted-Larsen, 
Nucl. Instrum. Methods Phys. Res., Sect. B 161-163, 1033 (2000).

${ }^{35}$ W. Wesch, C. S. Schnohr, P. Kluth, Z. S. Hussain, L. L. Araujo, R. Giulian, D. J. Sprouster, A. P. Byrne, and M. C. Ridgway, J. Phys. D: Appl. Phys. 42, 115402 (2009).

${ }^{36}$ M. D. Hou, S. Klaumünzer, and G. Schumacher, Phys. Rev. B 41, 1144 (1990)
${ }^{37}$ A. Colder, O. Marty, B. Canut, M. Levalois, P. Marie, X. Portier, S. M. M. Ramos, and M. Toulemonde, Nucl. Instrum. Methods Phys. Res., Sect. B 174, 491 (2001).

${ }^{38}$ A. Kamarou, W. Wesch, E. Wendler, A. Undisz, and M. Rettenmayr, Phys. Rev. B 78, 054111 (2008).

${ }^{39}$ A. Kamarou, W. Wesch, E. Wendler, A. Undisz, and M. Rettenmayr, Phys. Rev. B 73, 184107 (2006). 